\title{
Financiamento Público para a CT\&I e a Geração de Riqueza no Nível Subnacional: análise dos investimentos da Fapesc na década de 2010
}

\author{
Public Funding to ST\&I and to the Generation of Wealth at the \\ Subnational Level: analysis of Fapesc investments in the decade 2010
}

\author{
Illyushin Zaak Saraiva ${ }^{1}$ \\ Bruna do Amaral ${ }^{2}$ \\ Camilo Freddy Mendoza Morejon ${ }^{3}$ \\ Cláudia Crisóstimo ${ }^{2}$ \\ Paulo Rogério de Pinto Rodrigues ${ }^{2}$ \\ ${ }^{1}$ Instituto Federal Catarinense, Campus Luzerna, Luzerna, SC, Brasil \\ ${ }^{2}$ Universidade Estadual do Centro-Oeste, Campus Guarapuava, Garapuava, PR, Brasil \\ ${ }^{3}$ Universidade Estadual do Oeste do Paraná, Campus Toledo, Toledo, PR, Brasil
}

\begin{abstract}
Resumo
No cenário brasileiro de CT\&I, a maior parte do financiamento ainda se sustenta em investimentos públicos, realizados por meio de fundações de fomento estaduais e de órgãos federais. Entre os estados brasileiros, Santa Catarina aparece atualmente em $5^{\circ}$ lugar em termos de montantes investidos pelos governos estaduais e na mesma posição no que diz respeito ao número de patentes obtidas. O objetivo deste estudo é mensurar e analisar a taxa de realização de investimentos da Fapesc, órgão catarinense de fomento em CT\&I, e descrever seu histórico. A pesquisa é documental e foi realizada com dados coletados nos arquivos do Estado de Santa Catarina e tratados no Microsoft Excel. Os resultados estão apresentados em gráficos e em tabelas e por meio deles realiza-se a análise descritiva. Conclui-se que, embora a produtividade da Fapesc seja destaque no Brasil, o órgão está à mercê do não cumprimento do percentual constitucional de repasses do orçamento estadual, por isso, sugere-se que sejam realizados estudos similares sobre entidades estaduais de fomento para a CT\&I.
\end{abstract}

Palavras-chave: Políticas Públicas de CT\&I. Fomento à Inovação. Fapesc.

\begin{abstract}
In the Brazilian ST\&I scenario, most of the funding still relies on public investments, carried out through state promotion foundations and federal agencies. Among the brazilian states, Santa Catarina currently ranks 5 th in terms of state amounts invested, and the same position with regard to the number of patents obtained. The objective of this study is to measure and analyze the investment rate of Fapesc, Santa Catarina state's promotion agency, describing its historic. The research is documentary, over data collected in the archives of Santa Catarina State, treated in Microsoft Excel, constructing graphs and tables from which descriptive analysis is performed. It is concluded that, although the productivity of Fapesc is prominent in Brazil, the agency is at the mercy of the noncompliance with the constitutional percentage of transfers from the state budget, suggesting to the research similar studies on state entities of funding to ST\&I.
\end{abstract}

Keywords: Public Policies of ST\&I. Fostering of Innovation. Fapesc.

Área Tecnológica: Inovação e Desenvolvimento. Economia do Conhecimento. 


\section{Introdução}

Com o advento da chamada revolução tecnológica das telecomunicações e da informática a partir dos anos 1980, o desenvolvimento industrial e econômico atingiu um ritmo nunca antes verificado durante a história do sistema capitalista, com efeitos variados sobre as famílias, as empresas e os demais agentes econômicos, permitindo uma transformação acelerada nos modos de produção e de distribuição de bens e serviços (CORREA, 2010) e, sobretudo, na oferta mundial de novos produtos e bens de consumo de alto conteúdo tecnológico, invadindo - como no caso dos atuais smartphones e tablets, que têm apenas pouco mais que uma década de existência - os mercados mais longínquos e gerando competição global a níveis extremos. Isso provocou, inclusive, o desaparecimento de vetores econômicos outrora pujantes e ocasionou a ruína econômica de firmas/regiões antes caracterizadas pela riqueza (GALLAGHER; PARK, 2002), colocando a geração de riqueza e de emprego baseada no desenvolvimento de novas tecnologias, surgidos a partir de investimentos em CT\&I, no centro das políticas econômicas em grande parte dos países, a chamada Economia do Conhecimento.

Nesse sentido, recentes prospecções econômicas em profundidade, com acesso a dados de alcance geográfico em bases mundiais, demonstram que essas mudanças evolutivas ocorrerão num ritmo ainda cada vez mais veloz em indústrias dotadas de maior potencial de inovação, ritmo movido

[...] por (a) a natureza cumulativa da mudança tecnológica; (b) a natureza exponencial de tecnologias como a dos microchips, que dobraram em capacidade de processamento a cada dois anos por meio século; (c) a convergência de distintas tecnologias em novas combinações; (d) a emergência de "plataformas de plataformas" digitais - mais notavelmente a internet; e (e) a diminuição dos custos de implantação. (UNCTAD, 2018, p. xi, tradução livre)

Entre essas novas tecnologias de destaque, a Unctad listou em 2018 as que detêm maiores potenciais de retorno, sendo elas as tecnologias de Big Data; de Internet das Coisas; de Inteligência Artificial; de Impressão em 3D; de Biotecnologia; de Nanotecnologia; de Energias Renováveis; a tecnologia dos Drones; e, finalmente, a tecnologia de projeto, construção e lançamento de Minissatélites Customizados (UNCTAD, 2018); tecnologias situadas na fronteira do conhecimento, nas quais governos, Universidades, empresas e entidades associativas deveriam se focar.

Em um cenário de rápida transformação, governos e organismos internacionais passam a assumir postura ainda mais estratégica no fomento à competitividade, explorando potencialidades naturais e/ou culturais das diversas regiões e incentivando mudanças visando ao desenvolvimento tecnológico nos diversos níveis (KITUYI, 2018), com destaque para entidades internacionais, como a Unctad, que, em seu último relatório, não apenas traça as diretrizes para a redução da desigualdade por meio do desenvolvimento tecnológico, mas vai além, explicitando quais as metodologias e as políticas públicas a serem utilizadas para se atingir esse desenvolvimento (UNCTAD, 2018).

O papel do Estado assume em cada região uma maior ou menor importância, prospectando e regulamentando políticas, mas também - e principalmente - investindo recursos públicos em CT\&I por meio de várias modalidades de fomento voltadas para as Universidades, as empresas, certos órgãos públicos (como institutos de saúde pública), e mesmo para inventores indepen- 
dentes, sendo digna de registro, nesse sentido, a diferença entre os sistemas Europeu, Brasileiro e Norte-Americano de investimento público em P\&D (SQUEFF; ALVES; BARCELOS, 2017; DE NEGRI, 2017).

Tratando-se especificamente das particularidades do cenário brasileiro, os dados do Ministério da Ciência, Tecnologia, Inovações e Comunicações mostram que os dispêndios Brasileiros com P\&D em 2016 totalizaram $\mathrm{R} \$ 79,23$ bilhões, dos quais 47,62\% tiveram como origem instituições privadas e $52,38 \%$ ( $\mathrm{R} \$ 41,5$ bilhões) órgãos públicos, sendo $\mathrm{R} \$ 11,8$ bilhões de gastos estaduais e $\mathrm{R} \$ 21,1$ bilhões de gastos federais, entre outras fontes governamentais destacadas pelo Ministério, como algumas empresas estatais e autarquias da União, Estados e Municípios (BRASIL, 2018).

De Negri (2017) mostra ainda, com dados de 2012, que entre os investimentos de origem federal em CT\&I, destacam-se o Ministério da Ciência, Tecnologia e Inovação, com 36\% do total, e o Ministério da Educação, com 19\%, seguidos pelos Ministérios da Agricultura e da Saúde, então com $13 \%$ e $11 \%$ dos aportes federais para CT\&I, respectivamente.

Ainda a respeito das políticas públicas de investimento em $\mathrm{P} \& \mathrm{D}$ no Brasil, desta vez com foco no plano intranacional, estudos mostram correlação entre o PIB dos Estados brasileiros, seus investimentos públicos em P\&D e o número de patentes obtidas, este último considerado um indicador clássico do sistema de inovação, apesar da tradicional observação acerca do descolamento entre publicações científicas e patentes no Brasil (CAVALCANTI; BEMFICA, 2019).

Embora o percentual de investimento total dos Estados seja de apenas cerca de $56 \%$ diante do total de investimento federal em P\&D (BRASIL, 2018), há algum tempo observa-se que, em determinados Estados, como São Paulo, é o investimento estadual o grande fomentador de inovação científica e tecnológica (QUADROS et al., 2000), apontando-se ainda para certas vantagens em termos de descentralização e de gestão apresentadas pela rede estadual de agências de fomento em P\&D em relação aos órgãos nacionais. Segundo os autores, as agências estaduais

[...] de fomento têm um papel muito importante no financiamento da pesquisa científica. Elas canalizam recursos financeiros, grande parte a fundo perdido, para as atividades de pesquisa de universidades e institutos de pesquisa localizados no Estado. [...] O caráter concorrencial é uma característica central do financiamento dessas agências, estimulando as instituições que executam a pesquisa (universidades, institutos e empresas) a competir entre si na busca de maior excelência e produtividade acadêmica $e$ científica. (QUADROS et al., 2000, p. 129)

Este estudo, que não tem a pretensão de mensurar os resultados ou a eficiência do retorno dos investimentos públicos em inovação, nem por meio do número de artigos publicados em periódicos indexados, do número de pedidos de patentes concedidas, nem de quaisquer outros indicadores existentes (MENDONÇA et al., 2018), por se tratar de tarefa complexa que exigiria provavelmente dados indisponíveis no curto prazo, propõe-se apenas a investigar preliminarmente a eficiência na execução financeira de investimentos previstos por uma agência estadual de fomento à CT\&I.

O objetivo do trabalho, portanto, é mensurar a taxa de realização de investimentos da Fapesc, órgão catarinense de fomento em CT\&I, em relação aos montantes previstos legalmente, com o intuito de realizar análises de ordem qualiquantitativa e produzir conjuntos de dados $e$ informações que permitam futuras análises aprofundadas sobre os resultados desta agência. 
Também é feito um curto resgate do histórico institucional desta fundação, desde sua origem ainda nos anos 1980.

Parte-se do pressuposto de que, para além das razões de ordem contextual já apresentadas por estudos como o de Cavalcante (2013) acerca do descolamento entre orçamento público para Ciência, Tecnologia e Inovação no Brasil e os resultados econômicos obtidos, uma possível razão para esse descolamento seria a falta de eficiência na realização dos investimentos previstos na legislação estadual pelos órgãos públicos de fomento, seja por razões de gestão, seja pela falta de repasses.

O trabalho é constituído de cinco seções, sendo esta introdução, a metodologia, os resultados, as considerações finais e as referências.

\section{Metodologia}

Neste estudo, os dados quantitativos analisados são aqueles referentes ao período 2010/2018, em razão da disponibilidade de informações desse intervalo por órgãos públicos de Santa Catarina, coincidindo com as mudanças de caráter modernizador introduzidas na Fapesc a partir de 2011.

Segundo Quivy e Campenhoudt (2008), o método quantitativo usa três principais sistemáticas de coleta de informações, a saber: a) a pesquisa em forma oral ou escrita (entrevistas e questionários); b) a observação; e c) a análise de documentos. Todos os dados aqui utilizados são secundários, obtidos por meio de pesquisa documental, com foco especial nos indicadores de execução financeira da Fapesc que estão disponíveis nos diversos portais de internet da Secretaria de Estado do Desenvolvimento Sustentável (SDS), do Tribunal de Contas do Estado de Santa Catarina (TCE-SC), e da própria Fapesc, além de alguma literatura acerca da Gestão da Inovação e de Políticas Públicas de Inovação e Transferência de Tecnologia. Os dados para 2018 são do Portal da Transparência do Estado de Santa Catarina (SANTA CATARINA, 2019).

Os dados acerca da execução financeira da Fapesc foram analisados de forma eminentemente quantitativa, por meio de análise descritiva, utilizando-se o software Microsoft Excel 2013. Algumas das tabelas disponibilizadas nas diversas bases de dados e websites consultados, quando em formato de arquivo PDF, foram inicialmente convertidas para o formato Microsoft Word 2013, sendo posteriormente convertidas para o formato Excel, a partir do qual foram tratados os dados de acordo com as análises mais adequadas aos objetivos, compondo-se tabelas e gráficos, conforme o tipo de variável e o universo de análise.

\section{Resultados e Discussão}

Originalmente, a instituição hoje denominada Fapesc foi instituída em 1990, com o nome de Fundo Rotativo de Fomento à Pesquisa Científica e Tecnológica do Estado de Santa Catarina (FUNCITEC), tendo posteriormente recebido a sigla atual - Fapesc, significando naquele momento (2005) Fundação de Apoio à Pesquisa Científica e Tecnológica do Estado de Santa Catarina, ainda sem qualquer menção à inovação no nome do órgão (FAPESC, 2018). 
Em 1995, embora o Funcitec tivesse mantido a sua sigla, passou a ser chamado Fundação de Ciência e Tecnologia, e, naquele mesmo ano, a instituição começou a funcionar no edifício do Centro Empresarial para Laboração de Tecnologias Avançadas (CELTA), que era então a representação do Governo Estadual de Santa Catarina no conjunto ParqTec Alfa, criado para alavancar o recente Polo Tecnológico de Florianópolis, inicialmente desenhado para gerir uma incubadora de empresas e diversos empreendimentos nascentes, então sob orientação da Fundação Certi - Centros de Referência em Tecnologias Inovadoras do Estado de Santa Catarina (FAPESC, 2015).

A Figura 1 apresenta o diagrama com a linha do tempo simplificada da Fapesc.

Figura 1 - Principais momentos na evolução da FAPESC

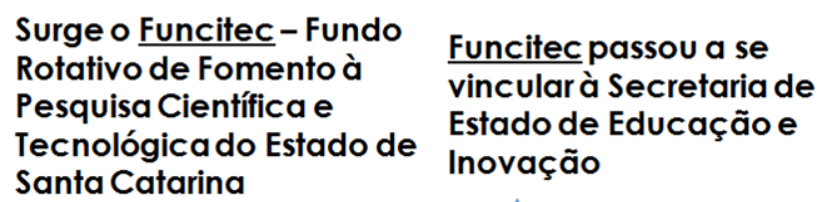

Santa Catarina

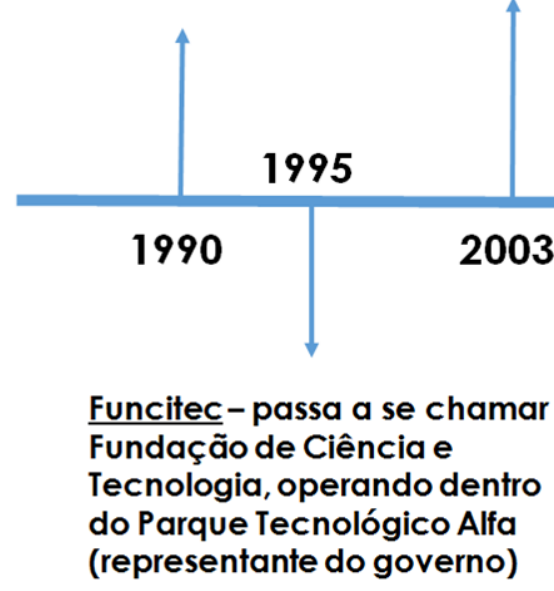

Foi a primeira entre as Fundações de Amparo à Pesquisa e Inovação no Brasil - FAPs a publicar chamada pública para o programa TECNOVA

Fonte: Elaborada pelos autores deste artigo com dados de Fapesc (2018b; 2015) e Gargioni (2015)

Foi em 2011 que, finalmente, o nome dessa agência pública de fomento passou a ser Fundação de Amparo à Pesquisa e Inovação do Estado de Santa Catarina, nome que permanece até os dias presentes, sendo que a inclusão do termo inovação no nome acompanhou a evolução da nomenclatura e do funcionamento das diversas Fundações Estaduais de Amparo à Pesquisa e Inovação (FAPs) do Brasil (GARGIONI, 2015).

Quanto à origem dos recursos da Fapesc, de acordo com a Constituição do Estado (SANTA CATARINA, 2017), a vinculação do orçamento estadual com a Ciência, Tecnologia e Inovação se dá segundo o percentual de $2 \%$ do orçamento total, que deve ser distribuído obrigatoriamente entre a Empresa de Pesquisa Agropecuária e Extensão Rural de Santa Catarina (EPAGRI) e a Fapesc.

A partir das mudanças profundas instauradas em 2011, a Fapesc passou a dividir os investimentos entre os seus quatro Macroprocessos Finalísticos principais, a saber, as atividades de fomento (1) à pesquisa; (2) à formação de recursos humanos - bolsas de estudo e de pesquisa; (3) à divulgação científica; e (4) à Inovação.

A seguir, no diagrama da Figura 2, apresenta-se uma sistematização simplificada das iniciativas de fomento da Fapesc, segundo cada Macroprocesso Finalístico. 
Figura 2 - Diagrama dos Macroprocessos Finalísticos da Fapesc

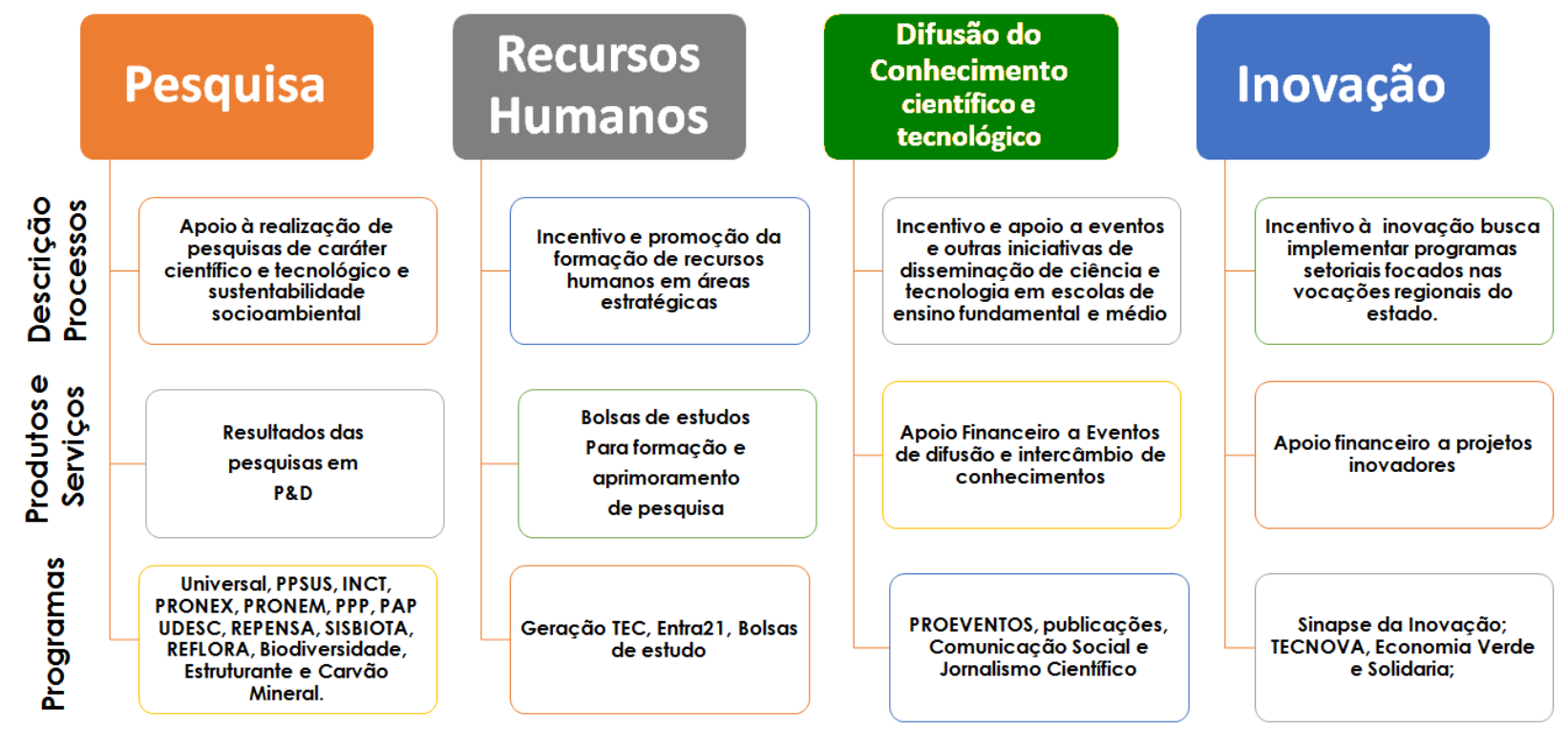

Fonte: Elaborada pelos autores deste artigo com dados da Fapesc (2017)

As iniciativas de fomento da Fapesc constituem-se de um grande conjunto de atividades realizadas em base estadual, sendo a maioria delas oferecidas na forma de editais e chamadas públicas para concorrência entre Pesquisadores, Grupos de Pesquisa ou Universidades e Instituições de Ensino Superior, garantindo a qualidade dos projetos aprovados e a lisura no processo.

Há, contudo, iniciativas específicas voltadas a investimentos setoriais, por exemplo, projetos ambientais e ações ligadas a comunidades indígenas, nas quais o investimento é feito pela Fapesc em conjunto com certos órgãos ou instituições determinadas previamente, como a Empresa de Pesquisa Agropecuária e Extensão Rural de Santa Catarina (EPAGRI) e a Universidade do Estado de Santa Catarina (UDESC).

Com as mudanças implementadas a partir de 2011, os resultados na eficiência da Fapesc passaram a aparecer já em 2012, quando a submissão dos projetos e a prestação de contas (ao final da duração dos projetos) passaram a ser permitidas via web, utilizando-se da Plataforma de CTI da Fapesc, o que trouxe grande agilidade na tramitação dos processos (FAPESC, 2015).

Em agosto de 2013 a Fapesc tornou-se a primeira entre as Fundações de Amparo à Pesquisa e Inovação no Brasil - FAPs a publicar chamada pública para o programa TECNOVA, que surgiu da parceria entre FINEP e FAPs (FAPESC, 2015).

E, em 2013, outro edital inédito foi lançado com bolsas para estágios pós-doutorais em empresas, com o propósito de iniciar a relação necessária entre a academia e o setor produtivo, pugnada no conceito da Tripla Hélice, com investimentos privados saltando de 9,3\% em 2011 para $14,4 \%$ do total da Fapesc em 2013, um aumento de mais de 50\% em apenas dois anos (GARGIONI, 2015). 
As informações apresentadas a seguir são fruto de análises sobre dados de pesquisa de ordem documental e bibliográfica, sendo que apenas estão disponíveis para consulta dados a partir do ano 2010 (relatórios publicados em 2011), sobre os quais se efetuou tratamento descritivo simples.

Com relação à consulta a dados de alta qualidade sobre o período imediatamente anterior ao aqui analisado (2002/2009), recomenda-se uma pesquisa específica sobre os dados compilados e apresentados no trabalho de Rezini (2010), especialmente a Tabela 10 (REZINI, 2010, p. 92).

Conforme já exposto, o artigo 193 da Constituição de Santa Catarina de 1989 (SANTA CATARINA, 2017) e a Lei da Inovação, Lei n. 14.328, de 2008 (SANTA CATARINA, 2008), obrigam o estado a destinar um mínimo de $2 \%$ da receita à pesquisa científica e tecnológica, $50 \%$ para a Empresa de Pesquisa Agropecuária e Extensão Rural (EPAGRI) de Santa Catarina e 50\% para a Fapesc (SANTA CATARINA, 2017).

O Gráfico 1 apresenta o total de repasses do governo estadual efetuados para a Fapesc ao longo dos anos de 2010/2018, com base em informações dos órgãos governamentais.

Gráfico 1 - Evolução dos repasses do Governo do Estado de Santa Catarina efetivados à Fapesc

\section{Repasses do Orçamento Estadual efetivados para a FAPESC - 2010/2018 (em R\$ Milhões) \\ Dados coletados em abril 2019 \\ Fontes: Relatório SBPC sobre a FAPESC e CTI em SC (SBPC, 2018); \\ Relatórios do Tribunal de Contas de SC (TCE-SC, 2011 a 2018), Portal da Transparência (SC, 2019)}

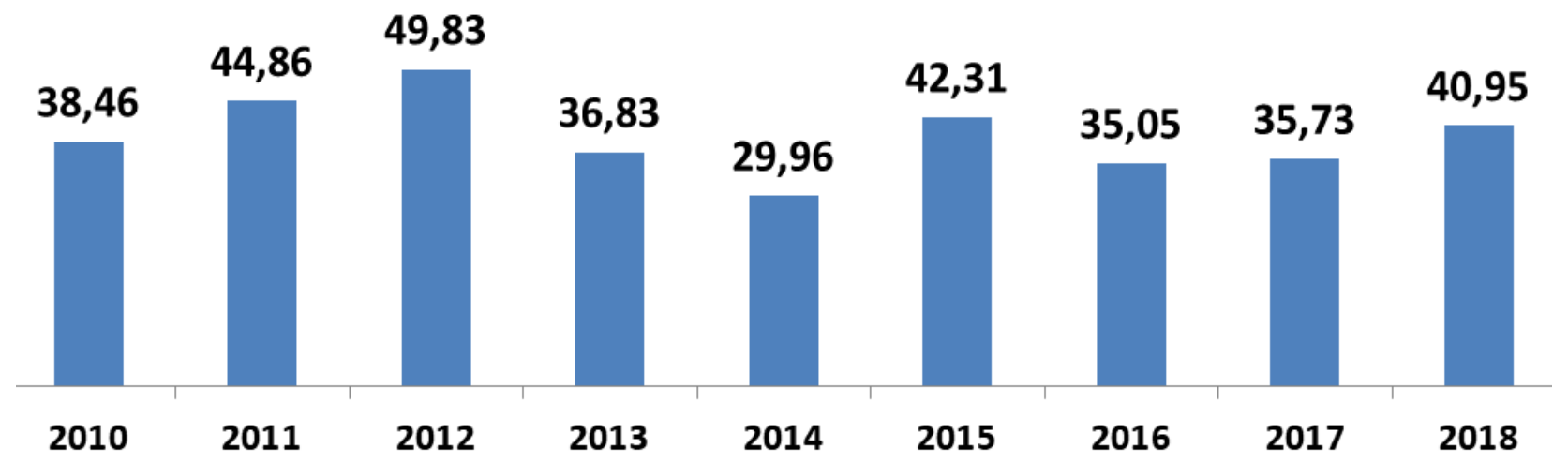

Fonte: Elaborado pelos autores deste artigo com dados de SBPC (2018); TCE-SC (2011; 2012, 2013; 2014; 2015, 2016; 2017; 2018) e Santa Catarina (2019)

Como é possível notar, os investimentos até 2012 apresentam tendência crescente e estável, sendo que, a partir de 2013, os valores passam por mudanças bruscas, relacionadas de alguma maneira à chegada dos efeitos da crise financeira internacional no Brasil e, sobretudo, à crise fiscal vivenciada no segundo mandato de Dilma Rousseff, nunca, porém, alcançando o montante investido no ano de 2012 , da ordem de $\mathrm{R} \$ 49,83$ milhões, que, até o momento, é o recorde em repasses para a fundação.

Outro dado significativo acerca do investimento em CT\&I pode ser visualizado no Gráfico 2, com o percentual do Orçamento Estadual destinado à Fapesc ao longo dos anos, que nunca atingiu o valor estipulado pela Constituição Estadual, de $1 \%$. 
Gráfico 2 - Percentual do Orçamento Estadual efetivamente investido na Fapesc

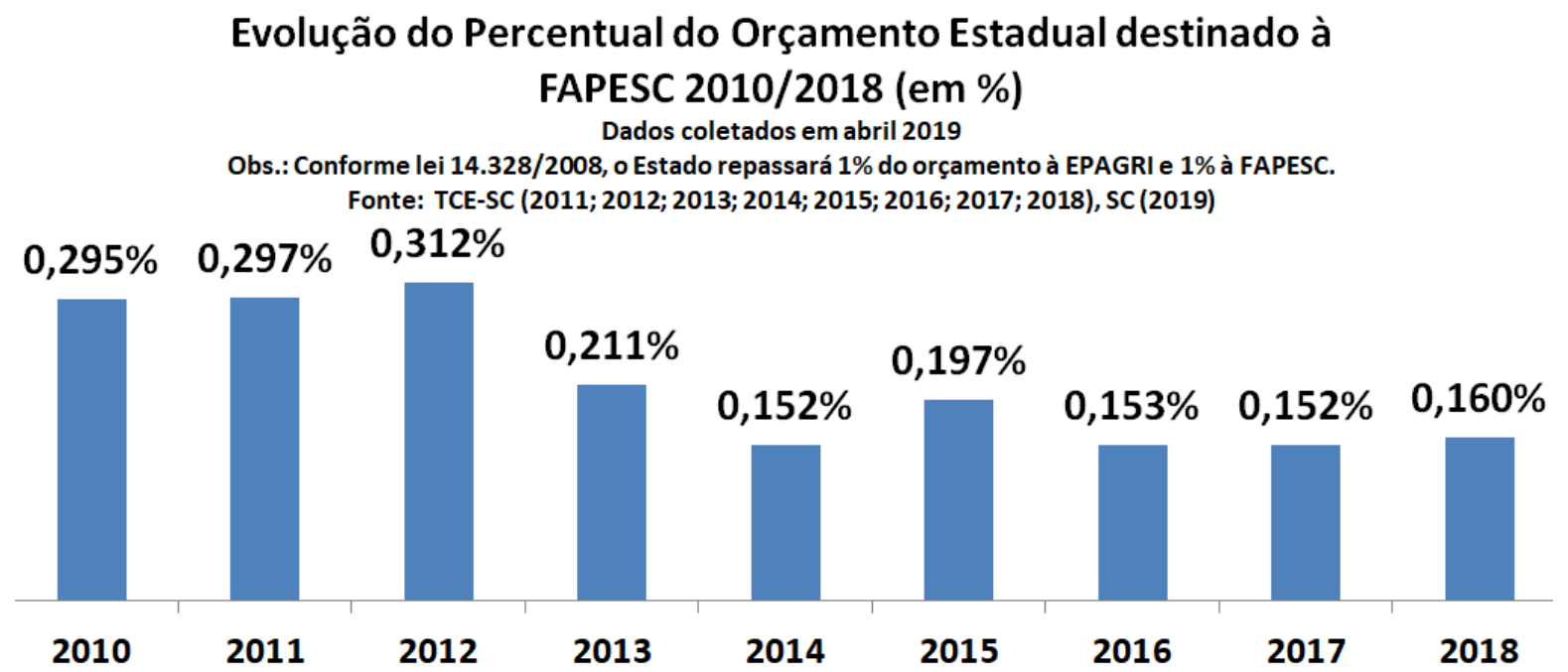

Fonte: Elaborado pelos autores deste artigo com dados de SBPC (2018), TCE-SC (2011; 2012, 2013; 2014; 2015, 2016; 2017; 2018) e Santa Catarina (2019)

Como se vê no Gráfico 2, durante o período aqui analisado, o governo do Estado de Santa Catarina nunca chegou a repassar à Fapesc o percentual de $1 \%$ do orçamento do estado, atingindo no máximo $0,31 \%$ em 2012, a partir de quando os percentuais baixaram significativamente, girando em torno dos 0,15\% de 2014 a 2018.

Com o propósito de perceber o quanto significaria em efetivo o montante total a ser repassado à Fapesc, caso o governo do estado cumprisse a normativa de 1\%, o Gráfico 3 traz os repasses efetivamente realizados versus a projeção de repasses previstos constitucionalmente, em milhões de reais.

Gráfico 3 - Percentual do Orçamento Estadual efetivamente investido na Fapesc

\section{Repasses do Orçamento Estadual efetivados para a FAPESC - 2010/2018, versus o percentual constitucional (em R\$ Milhões) \\ Dados coletados em abril 2019}

Obs.: Conforme lei 14.328/2008, o Estado repassará 1\% do orçamento à EPAGRI e 1\% à FAPESC. Fontes: RelaRelatório SBPC sobre a FAPESC e CTI em SC (SBPC, 2018);

Relatórios do Tribunal de Contas de SC (TCE-SC, 2011 a 2018), Portal da Transparência (SC, 2019)

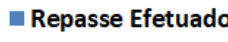

$1 \%$ Constitucional

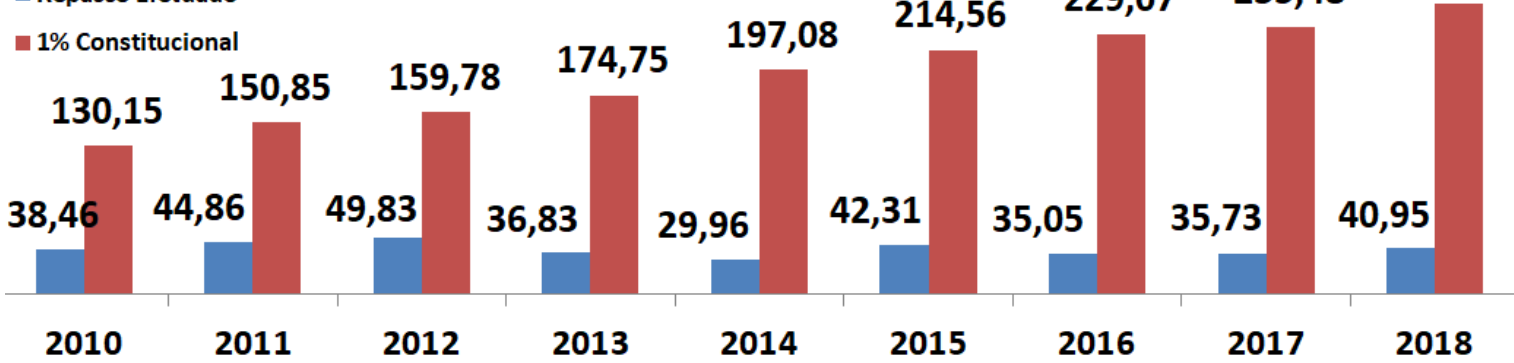

Fonte: Elaborado pelos autores deste artigo com dados de SBPC (2018), TCE-SC (2011; 2012, 2013; 2014; 2015, 2016; 2017; 2018) e Santa Catarina (2019)

Como é possível ver, o investimento em 2016 e em 2017 foi seis vezes menor que mínimo constitucional, e, com efeito, pode-se argumentar que o número de bolsas de iniciação científica, de mestrado e de doutorado poderia se multiplicar várias vezes caso o Governo do Estado 
repassasse à Fapesc o mínimo de 1\% estabelecido na Constituição do Estado, que, em 2018, teria representado o montante $\mathrm{R} \$ 256$ milhões.

Com um orçamento estadual que cresceu de $R \$ 13$ bilhões para mais de $R$ \$ 25,6 bilhões entre 2010 e 2018 (crescimento de 97\%), o valor total destinado à Fapesc subiu de R\$38 milhões para $\mathrm{R} \$ 41$ milhões no mesmo período (crescimento de apenas 6\%), demonstrando o efeito negativo da política de repasses efetivada pelo Estado, apesar das virtudes gerenciais da nova gestão da Fapesc adotadas a partir de 2011.

Importante registrar os apontamentos de Ramos (2018), ao analisar a política de investimentos em CT\&I do Governo de Santa Catarina, no sentido de que, a cada ano, deixa-se de aplicar cerca de $\mathrm{R} \$ 200$ milhões em todas as áreas do conhecimento por meio da Fapesc. Segundo o autor, "[...] não se trata de 'retirar' de outros setores para aplicar em CT\&I, mas sim de parar de transferir para outros setores, o que é devido constitucionalmente à CT\&I catarinense desde 1989" (RAMOS, 2018, último parágrafo).

Além dos repasses do Tesouro Estadual, a Fapesc também mantém convênios firmados com órgãos federais (FINEp, CNPQ, entre outros), a partir dos quais recebe financiamento em áreas e projetos específicos. No Gráfico 4, é possível notar o montante do financiamento anual da Fapesc, por fonte, com dados fornecidos diretamente pela fundação, não sendo informada a natureza da origem: 'Outras Fontes'.

Gráfico 4 - Fontes de financiamento da Fapesc
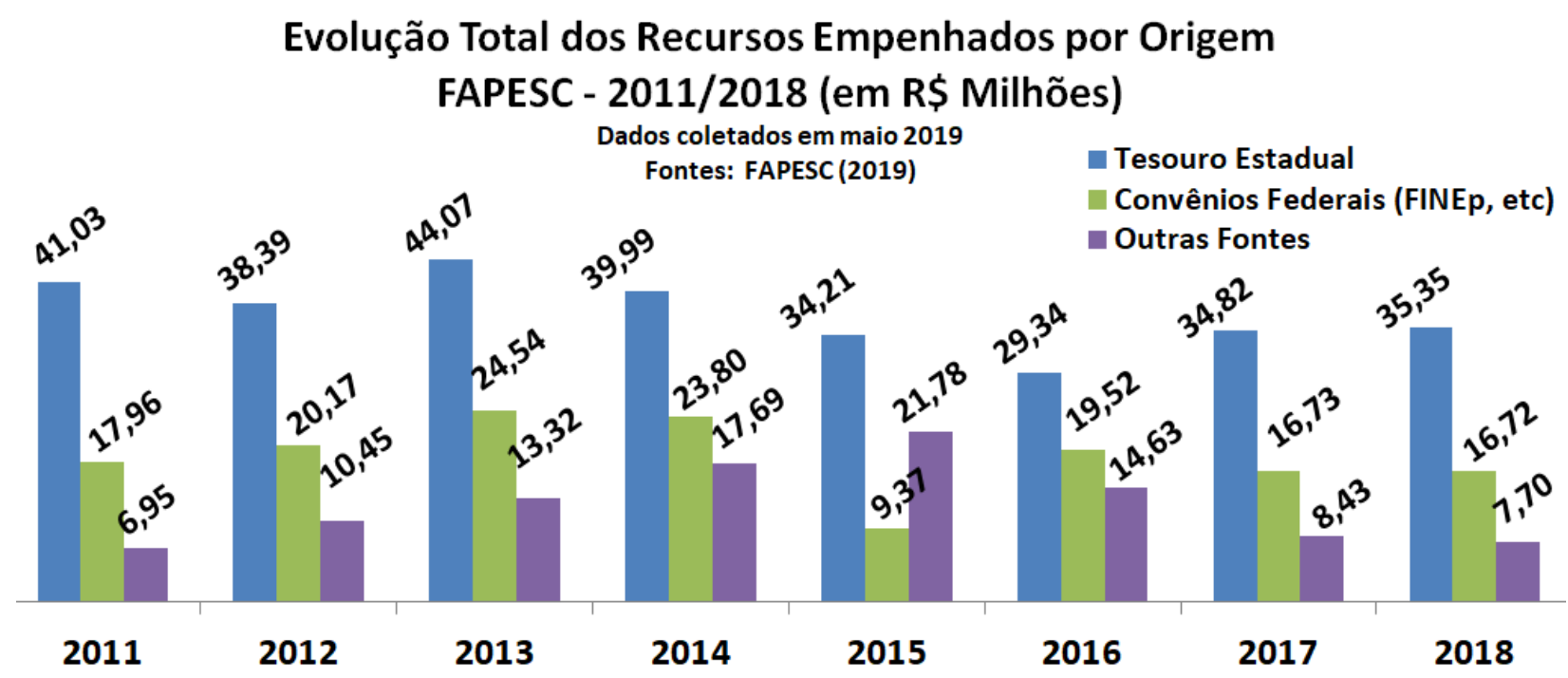

Fonte: Elaborado pelos autores deste artigo com dados da Fapesc (2019)

Esclareça-se inicialmente que a diferença entre os montantes da primeira coluna em cada ano (recursos empenhados do Tesouro Estadual) no Gráfico 4 (com valores obtidos diretamente da Fapesc) e os valores constantes do Gráfico 1 (com valores obtidos dos relatórios do Tribunal de Contas de Santa Catarina) se deve à natureza dos recursos apresentados, já que aqui se tratam dos valores da Programação Financeira, ou seja, dos valores da cota disponibilizada para fazer os empenhos.

Observa-se que os recursos federais empenhados apresentam uma evolução constante até 2013, momento a partir do qual eles começam a cair, sendo o pior desempenho o do ano de 2015 , quando apenas 9,37 milhões de reais em recursos federais foram empenhados. Interes- 
sante notar também que nesse mesmo ano de 2015 os recursos originários de outras fontes tiveram seu máximo, $\mathrm{R} \$ 21,78$ milhões.

Finalmente, analisa-se aqui brevemente a qualidade dos gastos da Fapesc, a partir da análise dos montantes investidos em cada Macroprocesso Finalístico da fundação, presentes no Gráfico 5 a seguir, com dados obtidos diretamente da Fapesc.

Gráfico 5 - Investimentos por Macroprocesso Finalístico

\section{Investimentos Realizados por Macroprocesso Finalístico FAPESC - 2011/2018 (em R\$ Milhões) \\ Dados coletados em maio 2019}

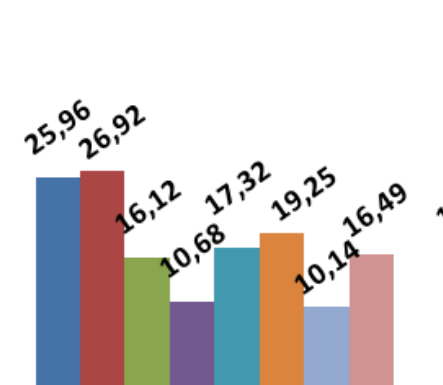

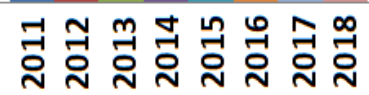

Pesquisa Científica
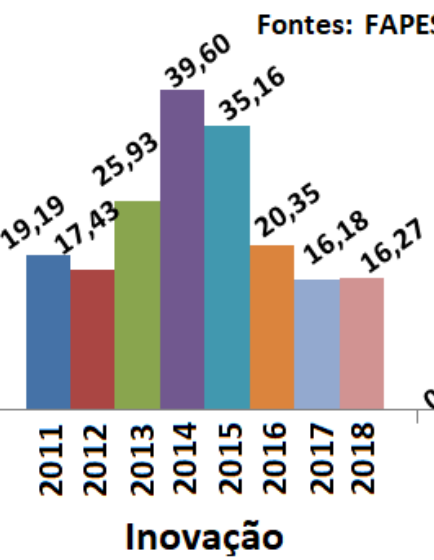

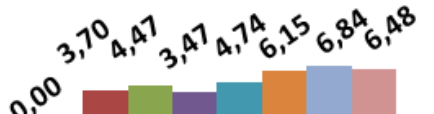

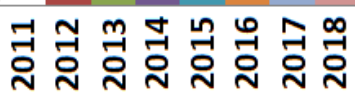
Recursos Humanos

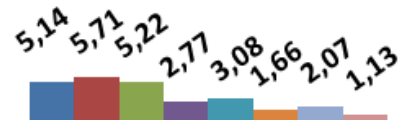

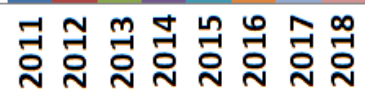
Difusão do Conhecimento

Fonte: Elaborado pelos autores deste artigo com dados da Fapesc (2019)

Acerca da análise dos dados do Gráfico 5, inicialmente é preciso esclarecer que a ausência de investimentos no Macroprocesso Recursos Humanos no ano de 2011 se deve ao fato de que até então não se consideravam os investimentos em RH como uma área, sendo os gastos com bolsas de Iniciação Científica, Mestrado e Doutorado categorizados na rubrica Difusão do Conhecimento.

Chama a atenção no gráfico o pico de investimentos em Inovação ocorrido nos anos 2013, 2014 e 2015. Também se observa que os montantes investidos em Inovação superaram aqueles destinados à Pesquisa Científica durante a maior parte do período analisado, sendo que esse excelente resultado pode em parte ser atribuído aos investimentos diretos no Programa Sinapse da Inovação, que de 2011 até 2017 aumentaram de R \$ 3,4 milhões para R\$ 10 milhões por cada edição, uma das marcas do pioneirismo da Fapesc, programa este que já foi levado a outros estados devido aos seus resultados em termos de empreendedorismo e geração de riqueza (FAPESC, 2018b); além de investimentos da própria Fapesc em outros programas, como o Pappe e o Tecnova.

Grosso modo, se percebe pelos dados do Gráfico 5 que os montantes destinados à Pesquisa Científica e à Inovação superam em muito os dos outros dois Macroprocessos, argumentando-se aqui que, dessa forma, uma das oportunidades para investimento, caso a Fapesc viesse num futuro próximo a ser contemplada com repasses obedecendo aos montantes constitucionais, seria justamente um aumento massivo na rubrica Recursos Humanos, podendo aumentar em muitas vezes o número de bolsas para estudantes engajados em projetos de CT\&I, além da realização de mais eventos de ciência e de inovação, e da publicação de mais estudos, por meio do investimento na rubrica Difusão do Conhecimento. 
Essa observação caminha na direção do que apontam especialistas acerca da necessidade de se remunerar mais e melhores pesquisadores no Brasil, já que são eles que executam a pesquisa - seja ela básica ou aplicada - e, para tanto, eles precisam ser recrutados, selecionados e remunerados; e o atual cenário limita enormemente a manutenção de jovens talentos na atividade de pesquisa, devido ao pequeno número de bolsas oferecidas e ao seu valor relativamente baixo (GALEMBECK, 2005).

Chega-se nesse momento à conclusão de que a Fapesc tem se destacado nacionalmente em termos da eficiência de sua Gestão e no caráter inovador de seus projetos de fomento à Inovação, tendo como exemplo principal o programa Sinapse da Inovação, concebido, gestado e implantado pela própria Fapesc, que premia a cada dois anos as 100 melhores ideias de negócio submetidas por milhares de empreendedores e de inventores individuais com $\mathrm{R} \$ 100$ mil em recursos para cada um dos vencedores, além de oferecer treinamento e mentoria a eles por meio do Sebrae-SC, e cujo sucesso inspirou a criação de programas similares no Paraná, no Espírito Santo e no Amazonas (FAPESC, 2018b).

Embora os investimentos financeiros realizados diretamente no Programa Sinapse não representem montante tão significativo em termos de recursos para inovação no cenário estadual, seus efeitos mais duradouros e, talvez, mais importantes em termos de inovação se devem à demanda qualificada que ele gerou para outros programas de investimentos em inovação, tendo como exemplo o Pappe Subvenção, programa da Finep que, em convênio com a Fapesc, investiu $\mathrm{R} \$ 12$ milhões em 116 projetos catarinenses durante seus sete anos de duração (FAPESC, 2016) e o Tecnova, programa da própria Fapesc com recursos da Finep que entre 2015 e 2018 concedeu $\mathrm{R} \$ 22,6$ milhões para 53 micro e pequenas empresas do estado (FAPESC, 2018b), entre outras ações.

A Fapesc também foi a primeira Fundação Estadual a adotar a submissão de projetos e a prestação de contas totalmente on-line, a partir de 2012, como fruto da adoção de novos paradigmas de inovação iniciados com as grandes mudanças de gestão em 2011.

E teve ainda participação direta na criação do Parque Tecnológico de Florianópolis, um dos 10 mais influentes do Brasil, a partir da construção e da instalação de sua sede dentro do Parque.

Observa-se, assim, no que diz respeito ao objetivo principal deste trabalho, que, apesar da qualidade da sua gestão no fomento à Ciência, Tecnologia e Inovação, a Fapesc não está imune à descontinuidade administrativa entre diferentes mandatos governamentais, nem ao descumprimento nos repasses financeiros legalmente previstos, sem que com isso se queira aqui e agora tecer quaisquer críticas de caráter proselitista ou ideológico, já que os governos têm suas prioridades e demandas, e o que se avalia é a atuação da agência de fomento.

Finalmente, considera-se que o objetivo deste modesto trabalho, que era realizar uma análise dos índices de realização dos investimentos financeiros da Fapesc em CT\&I, foi cumprido, na medida em que se analisou por meio de gráficos os dados quantitativos obtidos dos órgãos Estaduais acerca da evolução dos repasses recebidos e dos gastos realizados pela fundação entre 2010 e 2018, além de se apresentar um pequeno histórico de sua atuação. 


\section{Agradecimentos}

Registra-se o agradecimento à Ouvidoria do Estado de Santa Catarina e à Fapesc pela disponibilização dos dados utilizados na elaboração de alguns gráficos e figuras.

Também se registra o mais profundo agradecimento ao Instituto Federal Catarinense pela bolsa de afastamento para pós-graduação do primeiro autor, sem a qual este e outros trabalhos não poderiam ter sido concluídos.

\section{Referências}

BRASIL. Ministério da Ciência, Tecnologia, Inovações e Comunicações. Recursos Aplicados: Indicadores Consolidados, 2000-2016. Outubro de 2018. Disponível em: https://www.mctic.gov.br/ mctic/opencms/indicadores/detalhe/recursos_aplicados/indicadores_consolidados/2_1_3.html. Acesso em: 14 abr. 2019.

CAVAlCANTE, Luiz Ricardo. Consenso Difuso, Dissenso Confuso: Paradoxo das Políticas de Inovação no Brasil. IPEA: Texto para Discussão n. 1.867. 2013. Disponível em: https://www.econstor. eu/bitstream/10419/91358/1/768539404.pdf. Acesso em: 30 abr. 2019.

CAVALCANTI, André Marques; BEMFICA, Eduardo Andrade. Disseminação da Propriedade Intelectual como Estratégia para Políticas de Ciência, Tecnologia e Inovação: O caso do Sistema Pernambucano de Inovação (SPIN). Cadernos de Prospecção, Salvador, v. 12, n. 1, p. 15-30, março 2019. Disponível em: http://dx.doi.org/10.9771/cp.v12i1.27222. Acesso em: 30 abr. 2019.

CORREA, Henrique Luiz. Gestão de redes de suprimentos. São Paulo: Atlas, 2010.

DE NEGRI, Fernanda. Por uma nova geração de políticas de inovação no Brasil. In: TURCHI, Lenita Maria; MORAIS, J. Mauro. (org.). Políticas de apoio à inovação Tecnológica no Brasil: avanços recentes, limitações e propostas de ações. Brasília: IPEA, 2017. p. 25-46. Disponível: repositorio.ipea.gov.br/bitstream/11058/8125/1/Políticas\%20de\%20apoio\%20à\%20inovação\%20 tecnológica\%20no\%20Brasil.pdf. Acesso em: 22 nov. 2018.

FAPESC - FUNDAÇÃO DE AMPARO À PESQUISA E INOVAÇÃO DO ESTADO DE SANTA CATARINA. Histórico. Institucional, [S.l.], 2018a. Disponível em: www.fapesc.sc.gov.br/historico/. Acesso em: 16 nov. 2018.

FAPESC - FUNDAÇÃO DE AMPARO À PESQUISA E INOVAÇÃO DO ESTADO DE SANTA CATARINA. Fapesc faz Balanço dos 7 Anos do Programa PAPPE. 14 set. 2016. Disponível em: www.fapesc.sc.gov.br/fapesc-faz-balanco-dos-7-anos-do-programa-pappe/. Acesso em: 10 jan. 2019.

FAPESC - FUNDAÇÃO DE AMPARO À PESQUISA E INOVAÇÃO DO ESTADO DE SANTA CATARINA. Quatro Anos em Revista - Relatório de Atividades - 2011/2014. Florianópolis: Imprensa Oficial de Santa Catarina. 2015. 56p. Disponível em: www.fapesc.sc.gov.br/relatorio-deatividades/. Acesso em: 16 nov. 2018.

FAPESC - FUNDAÇÃO DE AMPARO À PESQUISA E INOVAÇÃO DO ESTADO DE SANTA CATARINA. Relatório de Atividades - 2011. Florianópolis: Imprensa Oficial de Santa Catarina. 2012. 52p. Disponível em: www.fapesc.sc.gov.br/relatorio-de-atividades/. Acesso em: 16 nov. 2018. 
FAPESC - FUNDAÇÃO DE AMPARO À PESQUISA E INOVAÇÃO DO ESTADO DE SANTA CATARINA. Relatório de Atividades - 2012. Florianópolis: Imprensa Oficial de Santa Catarina. 2013. 44p. Disponível em: www.fapesc.sc.gov.br/relatorio-de-atividades/. Acesso em: 16 nov. 2018.

FAPESC - FUNDAÇÃO DE AMPARO À PESQUISA E INOVAÇÃO DO ESTADO DE SANTA CATARINA. Relatório de Atividades - 2015-2018 - Balanço Social. Florianópolis: FAPESC. 2018b. 34p. Disponível em: www.fapesc.sc.gov.br/wp-content/uploads/2015/01/relatoriofapesc-1a30com-botao-compressed.pdf. Acesso em: 10 jan. 2019.

FAPESC - FUNDAÇÃO DE AMPARO À PESQUISA E INOVAÇÃO DO ESTADO DE SANTA CATARINA. Relatório de Gestão 2016. Florianópolis: FAPESC, 2017. 74p. Disponível em: www. fapesc.sc.gov.br/wp-content/uploads/2018/05/relatório-de-gestão-fapesc-final-2016_tcsc.pdf. Acesso em: 16 nov. 2018.

FAPESC - FUNDAÇÃO DE AMPARO À PESQUISA E INOVAÇÃO DO ESTADO DE SANTA CATARINA. Resposta à Ouvidoria 2011 a 2018. Planilha MS-Excel enviada aos autores em 13 maio 2019. (Não Publicada)

FAPESC - FUNDAÇÃO DE AMPARO À PESQUISA E INOVAÇÃO DO ESTADO DE SANTA CATARINA. Resumo de Atividades - 2013. Florianópolis: Imprensa Oficial de Santa Catarina, 2014. Disponível em: www.fapesc.sc.gov.br/relatorio-de-atividades/. Acesso em: 16 nov. 2018.

GALEMBECK, Fernando. Organização de pesquisa no Brasil: lições do passado, propostas para o futuro. Química Nova, [S.l.], v. 28, Suplemento, p. S52-S55, 2005. Disponível em: www.scielo. br/pdf/qn/v28s0/26776.pdf. Acesso em: 3 maio 2019. DOI: http://dx.doi.org/10.1590/S010040422005000700011.

GALLAGHER, Scott; PARK, Seung Ho. Innovation and competition in standard-based industries: a historical analysis of the US home video game market. IEEE Transactions on Engineering Management, [S.l.], v. 49, n. 1, p. 67-82, 2002. Disponível em: http://doi.org/10.1109/17.985749. Acesso em: 2 dez. 2018.

GARGIONI, Sérgio Luiz. Inovação na Prática. In: SANTA CATARINA (Estado). Quatro Anos em Revista: Relatório de Atividades Fapesc 2011/2014. Florianópolis: Imprensa Oficial de Santa Catarina. 2015. p. 30-36. Disponível em: www.fapesc.sc.gov.br/files/rel2011-14.pdf. Acesso em: 16 nov. 2018.

KITUYI, Mukhisa. Foreword. In: UNCTAD. Technology and Innovation Report 2018: Harnessing Frontier Technologies for Sustainable Development. Genebra, Suíça: United Nations Publication, 2018. ISBN 978-92-1-112925-0. Disponível em: https://unctad.org/en/PublicationsLibrary/tir2018_ en.pdf. Acesso em: 3 dez. 2018.

MENDONÇA, Valéria Melo et al. Indicadores Nacionais e Internacionais de Ciência, Tecnologia \& Inovação. Cadernos de Prospecção, Salvador, v. 11, n. 5, p. 1.293-1.304, dezembro, 2018. Disponível em: http://dx.doi.org/10.9771/cp.v12i5.27178. Acesso em: 16 mar. 2019.

QUADROS, Ruy et al. Força e fragilidade do sistema de inovação paulista. São Paulo em

Perspectiva, São Paulo, v. 14, n. 3, p. 124-141, jul. 2000. Disponível em: http://dx.doi.org/10.1590/ S0102-88392000000300018. Acesso em: $1^{\circ}$ maio 2019.

QUIVY, R.; CAMPENHOUDT, L. V. Manual de investigação em ciências sociais. 5. ed. Lisboa: Gradiva, 2008. 
RAMOS, André de Ávila. Quanto falta para que o estado de Santa Catarina cumpra sua Constituição e aplique 2\% do orçamento em CT\&I? Portal SBPC, on-line, 16 de agosto de 2018. Disponível em: portal.sbpcnet.org.br/noticias/quanto-falta-para-que-o-estado-de-santa-catarina-cumpra-suaconstituicao-e-aplique-2-do-orcamento-em-cti/. Acesso em: 16 nov. 2018.

REZINI, Débora Aparecida. Estudo das Ações Desenvolvidas por uma Instituição de Fomento à Pesquisa e Desenvolvimento: o caso Fapesc. 2010. 171p. Trabalho de Conclusão de Curso (Bacharelado em Economia) - Universidade Federal de Santa Catarina, Florianópolis, 2010. Disponível em: tcc.bu.ufsc.br/Economia292831. Acesso em: 30 abr. 2019.

SBPC - SOCIEDADE BRASILEIRA PARA O PROGRESSO DA CIÊNCIA. Cortes de recursos em ciência e tecnologia são debatidos na Alesc. Jornal da Ciência - Notícias da SBPC, Florianópolis, 2018. Disponível em: portal.sbpcnet.org.br/noticias/cortes-de-recursos-em-ciencia-e-tecnologia-saodebatidos-na-alesc/. Acesso em: 16 nov. 2018.

SANTA CATARINA. Constituição do Estado de Santa Catarina de 1989. Atualizada até a Emenda Constitucional n. 75, de 2017. Disponível em: http://leis.alesc.sc.gov.br/html/constituicao_ estadual_1989.html. Acesso em: 16 nov. 2018.

SANTA CATARINA. Lei n. 14.328, de 15 de janeiro de 2008. Dispõe sobre incentivos à pesquisa científica e tecnológica e à inovação no ambiente produtivo no Estado de Santa Catarina. Disponível em: http://www.sds.sc.gov.br/cecop/index.php/download/doc_download/22-lei-n-14-328-2008. Acesso em: 16 nov. 2018.

SANTA CATARINA. Despesa: Unidade Gestora Fundação de Amparo à Pesquisa e Inovação do Estado de Santa Catarina. Período: janeiro 2018 até dezembro 2018. Portal da Transparência do Poder Executivo. 2019. Disponível em: www.transparencia.sc.gov.br/documentos?v isao $=$ pagamento $\&$ unidadegestorafiltro $=270024 \&$ indicador $=0 \&$ tipoexecucaofiltro $=0 \&$ mesini $=01 \&$ anoini $=2018 \&$ mesfim $=12 \&$ anofim $=2018$. Acesso em 30 abr. 2019.

SQUEFF, Flávia de Holanda Schmidt; ALVES, Camila; BARCELOS, Catarina. Como a União Europeia Financia a Pesquisa? Radar IPEA, [S.I.], IPEA, n. 50, 2017. Disponível em: http:// repositorio.ipea.gov.br/bitstream/11058/7837/1/Radar_n50_como_a_União.pdf. Acesso em: 16 nov. 2018.

TCE-SC - TRIBUNAL DE CONTAS DO ESTADO DE SANTA CATARINA. Parecer Prévio sobre as Contas do Governo do Estado em 2012. 19 de dezembro de 2013. Disponível em: www.sef. sc.gov.br/arquivos_portal/relatorios/8/Parecer_Previo_do_TCE_2012.pdf. Acesso em: 16 nov. 2018.

TCE-SC - TRIBUNAL DE CONTAS DO ESTADO DE SANTA CATARINA. Relatório Técnico sobre as Contas do Governo do Estado, Exercício de 2010. 2011. Disponível em: www.tce.sc.gov.br/ sites/default/files/Relatorio_Tecnico_Contas_Gov_2010_Consolidado.pdf. Acesso em: 16 nov. 2018.

TCE-SC - TRIBUNAL DE CONTAS DO ESTADO DE SANTA CATARINA. Relatório Técnico sobre as Contas do Governo do Estado, Exercício de 2011. 2012. Disponível em: www.tce.sc.gov.br/ sites/default/files/Relatorio_Tecnico_Contas_Gov_2011_Consolidado_FINAL.pdf. Acesso em: 16 nov. 2018.

TCE-SC - TRIBUNAL DE CONTAS DO ESTADO DE SANTA CATARINA. Relatório Técnico sobre as Contas do Governo do Estado, Exercício de 2013. Em 3 de abril de 2014. Disponível em: www.tce.sc.gov.br/sites/default/files/Relatório\%20Técnico\%20Contas\%20Gov\%202013\%20 Consolidado\%2003-05-2014.pdf. Acesso em: 16 nov. 2018. 
TCE-SC - TRIBUNAL DE CONTAS DO ESTADO DE SANTA CATARINA. Relatório Técnico sobre as Contas Prestadas pelo Governo do Estado Relativas ao Exercício de 2014. 2015. Disponível em: www.tce.sc.gov.br/sites/default/files/RELATÓRIO\%20TÉCNICO\%20CONTAS\%20 DO\%20GOVERNO\%202014.pdf. Acesso em: 16 nov. 2018.

TCE-SC - TRIBUNAL DE CONTAS DO ESTADO DE SANTA CATARINA. Relatório Técnico sobre as Contas Prestadas pelo Governo do Estado Relativas ao Exercício de 2015. 2016. Disponível em: www.tce.sc.gov.br/sites/default/files/RelatorioTecnico_2015.pdf. Acesso em: 16 nov. 2018.

TCE-SC - TRIBUNAL DE CONTAS DO ESTADO DE SANTA CATARINA. Relatório Técnico sobre as Contas Prestadas pelo Governo do Estado Relativas ao Exercício de 2016. 2017. Disponível em: www.tce.sc.gov.br/sites/default/files/RELATÓRIO\%20TÉCNICO\%20CONTAS\%20 DO\%20GOVERNO\%202016.pdf. Acesso em: 16 nov. 2018.

TCE-SC - TRIBUNAL DE CONTAS DO ESTADO DE SANTA CATARINA. Relatório Técnico sobre as Contas Prestadas pelo Governo do Estado Relativas ao Exercício de 2017. 2018. Disponível em: www.tce.sc.gov.br/sites/default/files/RelatorioTecnico-2017.pdf. Acesso em: 16 nov. 2018.

UNCTAD - UNITED NATIONS CONFERENCE ON TRADE AND DEVELOPMENT. Technology and Innovation Report 2018: Harnessing Frontier Technologies for Sustainable Development. Genebra, Suíça: United Nations Publication, [2018]. ISBN 978-92-1-112925-0. Disponível em: https://unctad.org/en/PublicationsLibrary/tir2018_en.pdf. Acesso em: 30 abr. 2019.

\section{Sobre os Autores}

\section{Illyushin Zaak Saraiva}

E-mail: illyushin.saraiva@ifc.edu.br

Especialista em Educação Empreendedora pela Universidade Federal de São João del-Rei em 2014.

Endereço profissional: Instituto Federal Catarinense, Campus Luzerna, Rua Vigário Frei João, n. 550, Luzerna, SC. CEP: 89.609-000.

\section{Bruna do Amaral}

E-mail: bruna@solucoesimpar.com.br

Especialista em Gestão de Projetos pela Faculdade Sant'ana em 2016.

Endereço profissional: Universidade Estadual do Centro-Oeste, Agência de Inovação Tecnológica de Guarapuava.

Campus CEDETEG, Rua Simeão Varela de Sá, n. 3, Vila Carli, Guarapuava, PR. CEP: 85040-080.

\section{Camilo Freddy Mendoza Morejon}

E-mail: camilo_freddy@hotmail.com

Doutor em Engenharia Mecânica pela Universidade Federal do Rio de Janeiro em 2003.

Endereço profissional: Universidade Estadual do Oeste do Paraná, Centro de Engenharia e Ciências Exatas, Rua da Faculdade, n. 645, Jardim La Salle, Toledo, PR. CEP: 85903-000. 


\section{Cláudia Crisóstimo}

E-mail: crisostimoadv@gmail.com

Mestra em Direito pela Universidade Federal do Paraná em 2008.

Endereço profissional: Universidade Estadual do Centro-Oeste, Agência de Inovação Tecnológica de Guarapuava.

Campus CEDETEG, Rua Simeão Varela de Sá, n. 3, Vila Carli, Guarapuava, PR. CEP: 85040-080.

\section{Paulo Rogério de Pinto Rodrigues}

E-mail: prprodrigues@gmail.com

Doutor em Química pela Universidade de São Paulo em 1997.

Endereço profissional: Universidade Estadual do Centro-Oeste, Agência de Inovação Tecnológica de Guarapuava.

Campus CEDETEG, Rua Simeão Varela de Sá, n. 3, Vila Carli, Guarapuava, PR. CEP: 85040-080. 